Research Article

\title{
Cold Load and Storage Functional Backfill for Cooling Deep Mine
}

\author{
Mei Wang $\mathbb{D}^{1,2}$ Lang Liu ${ }^{1}$, ${ }^{1,2}$ Liu Chen, ${ }^{1,2}$ Xiaoyan Zhang, ${ }^{1,2}$ Bo Zhang, ${ }^{1,2}$ and Changfa Ji ${ }^{1,2}$ \\ ${ }^{1}$ School of Energy Engineering, Xi'an University of Science and Technology, Xi'an 710054, China \\ ${ }^{2}$ Key Laboratory of Western Mines and Hazard Prevention, Ministry of Education of China, \\ Xi'an University of Science and Technology, Xi'an 710054, China \\ Correspondence should be addressed to Lang Liu; liulang@xust.edu.cn
}

Received 23 November 2017; Accepted 29 January 2018; Published 5 July 2018

Academic Editor: Luigi Di Sarno

Copyright (c) 2018 Mei Wang et al. This is an open access article distributed under the Creative Commons Attribution License, which permits unrestricted use, distribution, and reproduction in any medium, provided the original work is properly cited.

Considering the deep mining heat-hazard problem, the concept and academic idea of cold load and storage (CLS) functional backfill applied on deep mine cooling was put forward. Firstly, according to characteristics of filling mining, a method of cooling stopes with CLS phase changing backfill which is made from the backfill material with CLS phase change material (PCM) was proposed. The working process, cooling physics, and the economic and safety benefits of CLS phase changing backfill were produced. Secondly, the theory system of cooling with CLS phase changing backfill was built. The theoretical basis of the key problems involved was investigated and analyzed which concluded heat transfer, fluid mechanics, and backfill mechanics. Lastly, the technology system of cooling with CLS phase changing backfill was established on the basis of the required technical assistance. It includes four parts: the backfill material design, the backfill material conveying way design, the stope temperature control scheme design, and the strength of cemented tailings backfill analysis. The idea of applying CLS phase changing backfill on cooling deep mine stopes and its theory and technology systems provide a scientific research and suitable development direction for deep mine cooling.

\section{Introduction}

As shallow mineral resources increasingly exhausted, the deep resource extraction has become normal. There are more than one hundred fifty $1000 \mathrm{~m}$-plus deep metal mines in the world at present. The deepest mines are in South Africa and Canada. There are 76 deep mines in South Africa with depth varying from 1524 to $4800 \mathrm{~m}$ and 32 mines in China with depth varying from 1000 to $1600 \mathrm{~m}$ [1]. The original rock temperature increases with depth. The average geothermal gradient is $2.5 \sim 4.0^{\circ} \mathrm{C} / 100 \mathrm{~m}$ [2], even the highest value is $7^{\circ} \mathrm{C} / 100 \mathrm{~m}$. Mponeng Gold Mine in South Africa reached $4000 \mathrm{~m}$ depth, and its geothermal temperature was up to $66^{\circ} \mathrm{C}$ [3]. The high geothermal temperature circumstance results in low efficiency of mining staff and high accident rate. According to the research of the influence of high mining temperature, when working face temperature raised from $16^{\circ} \mathrm{C}$ to $32^{\circ} \mathrm{C}$, the relative labor efficiencies decreased 55\%, and when this temperature raised from $29^{\circ} \mathrm{C}$ to $32^{\circ} \mathrm{C}$, the accident rate increased to $68.1 \%$ [4]. Meanwhile, high geothermal temperature also caused other threats, such as the softening of rock mass and the shortening of equipment service life and mineral combustion. Heat hazard has become an important problem which constraints the exploiting of the deep mine.

Facing heat hazard, there are three common cooling methods: ventilation, applying natural cold resource, and manual refrigeration. Increasing ventilation quantity is a cooling method which has longest history and widest application. However, it often cannot meet the cooling demands of deep mine with high temperature because of the restrains from inlet air temperature and velocity of working face. If the natural condition allows, applying underground cool water or storage ice form winter on cooling is an economic way. He and coworkers $[5,6]$ proposed the HEMS cooling system by using mine inflow as the extracted cold energy resource. It used the refrigeration system to extract cold from mine inflow and supply to air conditioner 
underground. $\mathrm{Bu}$ et al. [7] designed the ice making system which took advantages of the cold temperature in winter. Ice was produced in winter and stored in ice storage device and then used in summer. Shi et al. [8] proposed a spray ice making system and analyzed the feasibility of this method applying on mine cooling. Using natural cold energy is an economic and environmental method, but it had a limited application range because of the constraints of natural condition. The vast majority of mine adopted the cooling methods which are similar to the ground cooling. There are two typical systems: refrigeration equipment set in ground and underground. Though the chiller locations of the two types are different, there are some similarities. First, the cold load fluid pipe need to be laid, and the length of this pipe in the equipment centralized on ground type could be thousand meters. Second, some cooling equipment was set underground, especially the equipment centralized in underground type. The construction and management is complex, and the heat is difficult to exclude. The great investment of equipment and pipes, the complex construction and management, and the high running costs are the common problems in mine cooling engineering.

The implementation of the mine cooling techniques effectively mitigates the heat-hazard problem but also leads to the great increase of investment and energy consumption. According to some statistics, the power consumption of mine cooling system is about a quarter of the total mine power consumption [9]. In order to save energy and reduce contaminant, innovation of the cooling method based on exploiting characteristics could be a new way. GhoreishiMadiseh et al. [10] proposed a new method of using backfill to absorb underground heat to realize temperature control of stopes and geothermal energy utilization. The heat transfer model of extracting heat from underground by using the heat exchange pipe laid in backfill was built by numerical simulation and experimental research. Considering the cost of investment, this method is suitable for the case with high geothermal temperature and valuable in geothermal energy exploitation. For the lower geothermal temperature cases which are not suitable for geothermal energy exploitation, adding the cooling function to filling material and cooling stopes with functional backfill is an appropriate solution. Therefore the concept of cold load and storage (CLS) backfill cooling was proposed in this research.

\section{Cold Load and Storage (CLS) Functional Backfill}

2.1. The Conventional Cold Load and Storage Medium Used in Mine Cooling. The role of cold load and storage medium is transferring and storing cold from refrigerator to air conditioner. In the conventional mining cooling system, water, ice, and glycol solution were applied as cold load and storage medium usually. The system using water as coolant produced low-temperature chilled water by the refrigeration unit and then was pumped to air cooler exchanging heat with air. The cool wind was transported to working face to cool stopes. The system using ice as coolant produced ice cube, flake ice, or ice slurry by refrigerator on the ground.
And the ice was transported to the melt pool underground by wind or water power. When the ice was melt to cold water, it was surveyed to the working face, applying to air cooler or spray thrower for cooling stopes. The system using glycol solution as coolant produced low-temperature glycol solution by refrigerator, and then it was surveyed to heat exchanger underground to cool the water. The cold water was transported to terminal air cooler for cooling air. All the above cooling mediums were transported by a series of independent cooling system. Considering the energy saving and contaminant reduction, seeking a new coolant medium which combined with the mining method is a breakthrough way of mine cooling.

\subsection{Basic Contempt of CLS Functional Backfill. Filling} mining is one of the common mining methods. The backfill material was surveyed to gob and formed to backfill as mine working faces propelled. The volume could be hundreds of thousands of cubic meters. For the deep mine with serious heat-hazard problem and applying filling method, the huge backfill could be applied sufficiently as cooling material. By adding the CLS medium to the backfill material, a new CLS functional backfill material was created. The material was transported by backfill material survey pipe to gobs, and the stopes nearby were cooled through ground or wall's radiant cooling. This new cooling method leaves out the coolant transportation system and air conditioner terminal units.

\subsection{Cooling Process and Period of CLS Functional Backfill.} Taking the upward sublevel filling method as an example, as Figure 1 shows, when the CLS functional backfill material was surveyed to gobs, the heat was absorbed from stopes nearby and above. In the preliminary stage of cooling, the cooling potential of CLS functional backfill was large and was named period I (Figure 1(a)). When the cold energy released a lot to the stope, the cooling potential decreased and was named period II (Figure 1(b)). When the stope nearby above was exploited and gob was formatted completely, the cooling task of CLS backfill accomplished and the heat transfer between the stope and the backfill finished. As Figure 1(c) shows, this stage was named period III. Then the new gob was filled with CLS backfill material and a new period of cooling began.

2.4. CLS Functional Backfill Material. CLS functional backfill material is based on solid waste of mining, added with some phase change material (PCM) which has the cold load and storage ability and some cementitious material.

Sensible heat transfer and latent heat transfer are the two heat absorption types. The latent heat energy produced by phase change is $5 \sim 14$ times of sensible heat under the same volume condition [11]. PCM is very suitable for choosing as cold load and storage material by the advantage of its high cold storage density. CLS functional phase change backfill was made by mixing the PCM with conventional backfill material. The common PCM for cold storage consists of 


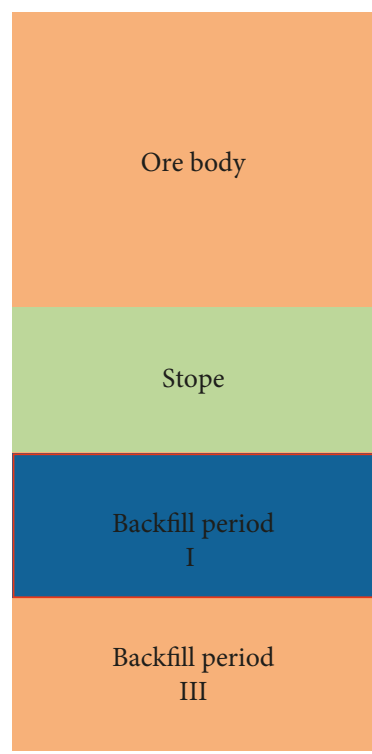

(a)

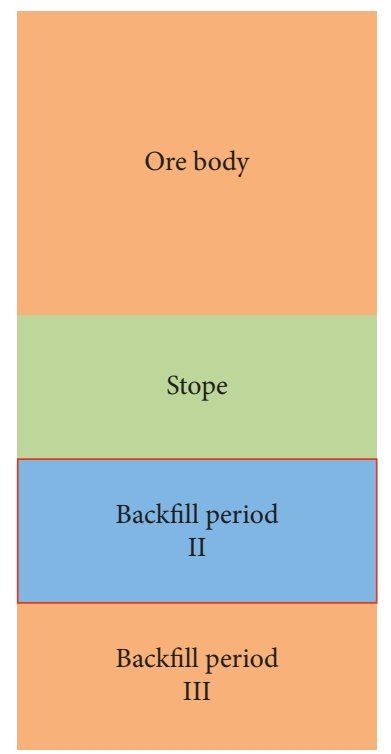

(b)

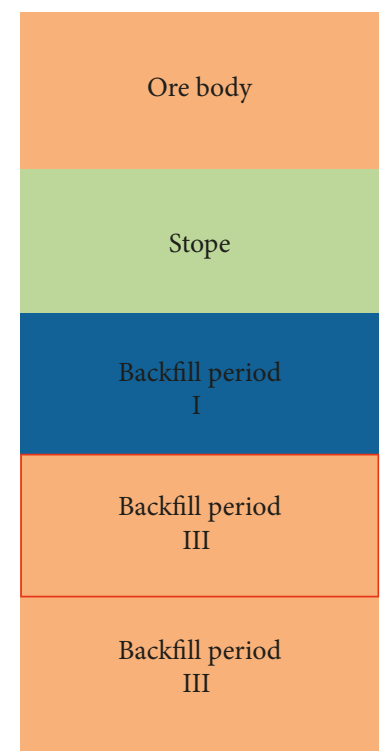

(c)

FIGURE 1: Cooling period of CLS backfill applied in upward sublevel filling method. (a) Preliminary period, (b) interim period, and (c) final period.

TABLE 1: Conventional cold storage PCM.

\begin{tabular}{lccc}
\hline Name of PCM & $\begin{array}{c}\text { Phase change } \\
\text { temperature }\left({ }^{\circ} \mathrm{C}\right)\end{array}$ & $\begin{array}{c}\text { Latent heat of } \\
\text { fusion }(\mathrm{kJ} / \mathrm{kg})\end{array}$ & Reference \\
\hline Ice & 0 & 335 & {$[12]$} \\
$\mathrm{Na}_{2} \mathrm{SO}_{4} \cdot 10 \mathrm{H}_{2} \mathrm{O}$ & 32 & 249 & {$[13]$} \\
$\mathrm{Na}_{2} \mathrm{SO}_{4} \cdot 10 \mathrm{H}_{2} \mathrm{O}$ & 8 & - & {$[14]$} \\
$\left(\mathrm{adding} \mathrm{NH}_{4} \mathrm{CL}\right)$ & 36 & 265 & {$[13]$} \\
$\mathrm{Na}_{2} \mathrm{HPO}_{4} \cdot 12 \mathrm{H}_{2} \mathrm{O}$ & $29 \sim 39$ & 174 & {$[13]$} \\
$\mathrm{CaCl}_{2} \cdot 6 \mathrm{H}_{2} \mathrm{O}$ & $-13 \sim-11$ & 187 & {$[15]$} \\
$\mathrm{Glycol}^{\text {Amino ethanol }}$ & $6 \sim 9.2$ & $155 \sim 196$ & {$[16]$} \\
aqueous solution & 9.5 & 157 & {$[17]$} \\
Paraffin oil/water & & &
\end{tabular}

organic and inorganic types (Table 1). The organic type PCM mainly includes pure substance (e.g., ice) and eutectic salt (e.g., $\mathrm{Na}_{2} \mathrm{SO}_{4} \cdot 10 \mathrm{H}_{2} \mathrm{O}$ ). It is widely used with mature technology; however, it has a salient phenomenon of super cooling and phase separation. The inorganic-type PCM mainly includes single component material (e.g., glycol) and eutectic mixture (e.g., paraffin oil). It has a wide phase change temperature range and good chemical properties but a lower cold storage density. The phase change material which has good performance on thermodynamic property (suitable phase change temperature, high latent heat, high heat capacity, and high heat conductivity), chemical property (good stability, noncorrosion, nonburning, nontoxic, and nonpolluting), and economic efficiency (cheap in material and charging cold) should be chosen as cold load and storage material.

The proportion of CLS functional backfill material should be determined by the consideration of flow characteristic, rheology property, strength characteristic, and cold supply capacity.
2.5. Advantages. Comparing with the conventional mine cooling method, the new method of applying CLS functional backfill on cooling has the following obvious advantages:

(i) No special pipes surveying cold load medium need to be laid. The transportation system of filling slurry takes the task of surveying cold load medium.

(ii) No air-conditioning equipment in the mine needs to be arranged. The radiant cooling was applied by the floor or wall of stopes.

(iii) No complex heat transfer between air-conditioning equipment exists, and the energy efficiency is high.

(iv) For the deposits with spontaneous combustion property, the whole low-temperature environment created by CLS functional backfill is beneficial in decreasing fire probability and enhancing mining security.

These advantages could sharply decrease the investment and running costs of mining cooling, reduce the construction and management of workload, and lower the fire probability. The CLS functional backfill cooling method has significant economic benefit and safety effect.

\section{Theories of CLS Functional Backfill Cooling}

3.1. Heat Transfer. Heat transfer (heat conduction, heat convection, and radiation) was driven by the temperature difference between CLS functional backfill and the surroundings. Figure 2 shows the total thermal equilibrium which consists of six terms of heat: $Q_{\mathrm{f}, \mathrm{l}}$, latent cold produced by PCM fusion in the CLS functional backfill; $Q_{\mathrm{f}, \mathrm{s}}$, sensible cold produced by low-temperature CLS functional backfill; $Q_{\text {cond }}$ and $Q_{\text {rad }}$, heat conduction and radiation from ore body to CLS functional backfill; $Q_{\text {conv }}$, heat convection from 


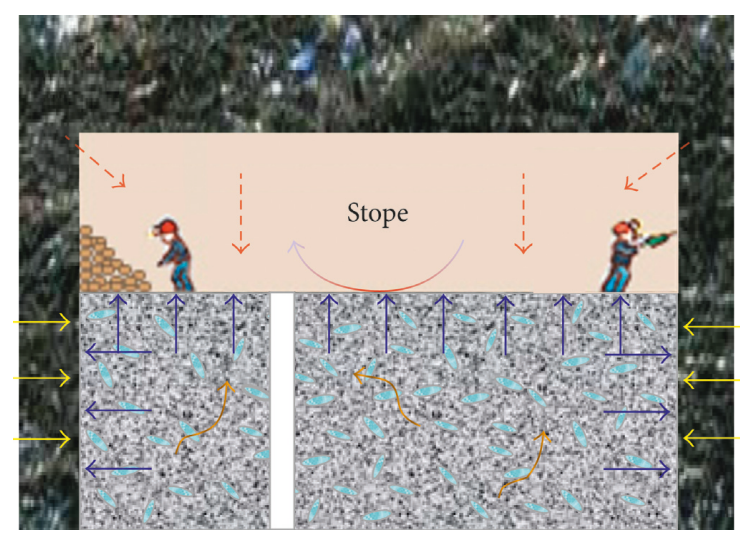

$$
\begin{array}{lll}
\longrightarrow \text { Discharged cold }\left(Q_{\mathrm{f}, 1}+Q_{\mathrm{f}, \mathrm{s}}\right) & \longrightarrow \text { Hydration heat }\left(Q_{\mathrm{h}}\right) \\
& \text { Conductive heat }\left(Q_{\text {cond }}\right) & \text { Ore body } \\
-\rightarrow \text { Radiant heat }\left(Q_{\mathrm{rad}}\right) & & \text { Functional backfill } \\
\text { Convective heat }\left(Q_{\text {conv }}\right) & \square \text { Sth iced particles }
\end{array}
$$

Figure 2: The total thermal equilibrium diagram of CLS phase change backfill cooling.

stopes to CLS functional backfill; and $Q_{\mathrm{h}}$, hydration heat produced by hydration reaction in the CLS functional backfill.

According to the first law of thermodynamics, the total thermal equilibrium equation was given:

$$
Q_{\mathrm{f}, \mathrm{l}}+Q_{\mathrm{f}, \mathrm{s}}=Q_{\text {cond }}+Q_{\text {conv }}+Q_{\mathrm{rad}}+Q_{\mathrm{h}} \text {. }
$$

With the cold releasing from CLS functional backfill, PCM fused and the temperature difference between backfill and surroundings diminished gradually. At last, the backfill temperature is consistent with surroundings and the cooling process finished. So the cooling process is a special process of cooling capacity decayed gradually. The cooling period and the decaying characteristics are the critical factors which influences on stope cooling effect. Equation (1) was taken a the derivative with respect to time to get (2) which expressed the gradient of releasing cold of CLS backfill to time.

$$
\frac{d Q_{\mathrm{f}, 1}}{d t}+\frac{d Q_{\mathrm{f}, \mathrm{s}}}{d t}=\frac{d \mathrm{Q}_{\text {cond }}}{d t}+\frac{d \mathrm{Q}_{\text {conv }}}{d t}+\frac{d \mathrm{Q}_{\mathrm{rad}}}{d t}+\frac{d \mathrm{Q}_{\mathrm{h}}}{d t} .
$$

The cooling process decaying characteristics of CLS functional backfill was calculated by studying the laws of heat transfer terms varying with time. The heat absorbed from surroundings through heat conduction, convection, and radiation depended on temperature difference between fillings and surroundings. As the temperature difference reduced, the heat transfer rate decreased. The hydration heat release rate related to the composition, hydration time, and temperature. According to the different composition, corresponding hydration products generated and the hydration heat released with the progress of hydration reaction. In particular, the hydration temperature is significant in this case. Owing to the low temperature of CLS filling slurry, the hydration reaction was restrained at the preliminary period obviously.

\subsection{Fluid Mechanics}

3.2.1. CLS Filling Slurry Transportation. CLS functional filling slurry composed of waste rock and tailings, PCM particles (take ice particles for instance following) and water. The diameter of solid particles is large, and the volume ratio is high; therefore, the filling slurry should be regarded as non-Newton fluid. According to the fluid regime supposed by Doron and Barnea [18] which researched water and sand two phase flow, there are three fluid regimes: static bed, fluidized bed, and fully suspended bed. When the average velocity of CLS filling slurry is large, strong turbulence flow effect resulted in a large component of pulse velocity which is perpendicular to the flow direction. Consequently, the force acting on the solid particles overcame the gravity of the solid particles which formed the fully suspended flow regime. When the flow speed decreased, the turbulence decreased which results in the phenomenon of waste rock and tailings flow in the bottom of the pipe and ice particles flow in the top of the pipes owing to the different densities. Thus the fluidized bed formed. When the flow speed decreased further, the static bed was formed which has the distribution of the ice particles in the top and the waste rock and tailings in the bottom (Figure 3).

The critical velocity and pressure drop researches of CLS filling slurry depend on the conventional filling slurry and ice slurry, on account of which the CLS filling slurry components are the conventional filling slurry and ice slurry (Table 2). However, the unique flow mechanism characteristics of CLS filling slurry should be noted: (1) The flow is accompanied by phase change which resulted in the gradient of slurry changed along the path, and therefore, the flow characteristics changed. (2) The hydration speed changed because of the rising temperature of slurry which absorbed heat from surroundings and the increasing water ratio caused by ice melting in the transportation process. So the gradient of slurry and flow characteristics changed. (3) The diameter of waste rock and tailings changed caused by hydration and the diameter of ice particles changed by melting and aggregation influenced the flow characteristics and even caused the pipe blocking.

In the storage or transportation process of ice, the ice particle size evolution behavior of nucleation and growth, aggregation, and fragmentation influenced the diameter of ice particles to change a lot from tens of to hundreds of micrometers which influenced the flow and heat transfer characteristics. Researchers have done a lot of work based on crystallization kinetic. Hansen et al. [25] indicated that the ice particle size changed in the ice storage tank even on the condition of thermal equilibrium. Under the GibbsThomson effect and Ostwald ripening effect, the small ice particles melt and get smaller, while the big particles grow and get bigger. Pronk et al. [26, 27] concluded that the distribution of ice particles changed because of the abrasion, aggregation, and Ostwald ripening effect. Especially the Ostwald ripening effect is the main reason of the ice particles growth. Furthermore, the influences of solution concentration and type on ripening were studied. Kanetoshi and Ken [28] introduced a set of measurement to detect 


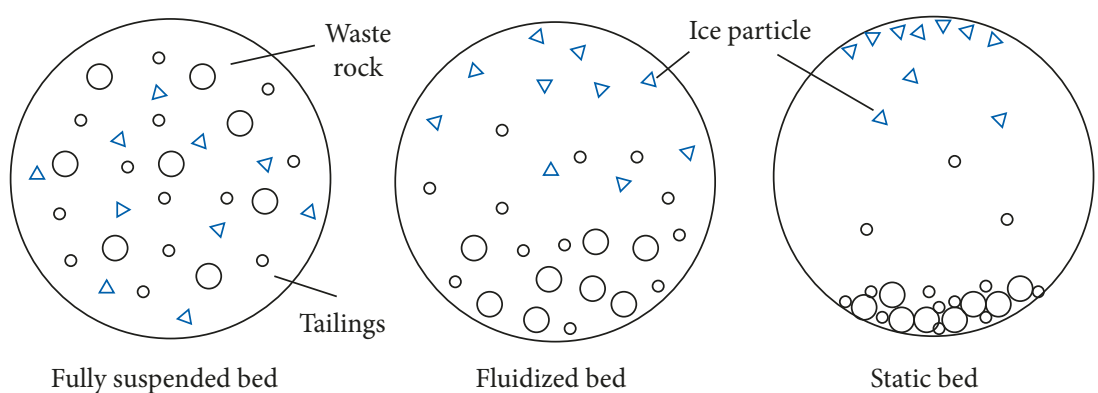

FIgURE 3: Three flow regime of CLS backfill slurry.

TABLE 2: The critical velocity formulas of slurry.

\begin{tabular}{|c|c|c|}
\hline Researchers & Formulas & Application \\
\hline $\begin{array}{l}\text { Durand } \\
{[19,20]}\end{array}$ & $F_{L} \sqrt{2 g D(1-S)}$ & $\begin{array}{c}\text { Filling } \\
\text { slurry, ice } \\
\text { slurry }\end{array}$ \\
\hline Knorroz [21] & $0.85(0.35+1.36 \sqrt[3]{C D}) \beta$ & Ice slurry \\
\hline $\begin{array}{l}\text { Guilpart } \\
\text { and } \\
\text { Fournasion } \\
{[22]}\end{array}$ & $2.8 \sqrt{g D(1-S)}$ & Ice slurry \\
\hline $\begin{array}{l}\text { Bechtel } \\
\text { Company } \\
{[23]}\end{array}$ & $K\left(\sqrt{\left(\rho_{S}-\rho\right) / \rho}\right) D^{1 / 3}\left(d_{95} / \eta\right)^{1 / 4} e^{\left(1+42 C_{V}\right)}$ & $\begin{array}{l}\text { Filling } \\
\text { slurry }\end{array}$ \\
\hline Fei [24] & {$\left[\left(2 g D S_{V}\left(\rho_{s}-\rho_{m}\right)\right) /\left(e_{s} f_{m} \rho_{m}\right)\right]^{1 / 3}$} & $\begin{array}{l}\text { Filling } \\
\text { slurry }\end{array}$ \\
\hline
\end{tabular}

aggregation by the electrical conductivity difference between ice and water. The experiment results showed that the bigger clump of ice was formed by aggregating the smaller ice particles and the low concentration of additives could restrain the aggregation. $\mathrm{Xu}$ et al. [29] developed the population balance model to study what factors influence the ice crystal distribution characteristics and its evolution during the process of ice slurry storage. The results indicated that with the decrease of the mass fraction of the additive solution, the growth rate of ice crystal size decreases. The lower IPF and higher dissipation of ice slurry slow the growth of ice crystal size in ice slurry solution. These researches established the foundation for the determination of ice particle diameter.

3.2.2. CLS Backfill Seepage. Some of water which consists of water in slurry and water changed from ice participated in hydration and the rest of water became the seepage. The seepage characteristics of CLS backfill is the basis of determination of drainage time, analysis of backfill strength, and the plan of continuous work. Mao et al. [30] studied the seepage law of upward sublevel hydraulic backfill. The mathematical model of dewatering time was established on the parameters of permeability coefficient, specific storage coefficient, and thickness. The dewatering coefficient was calculated, and the dewatering coefficient curves of different parameters were obtained. Wu et al. [31] coupled the hydraulic equations and thermodynamic equations, applying
COMSOL Multiphysics software and experiments to solve the seepage problem of cemented tailings backfill. The research indicated that with the higher initial temperature and curing temperature and the higher cement-sand mass ratio, the water permeability decreased. The analysis of initial temperature has significance for the research of CLS backfill. With higher temperature, the more hydration products were generated because hydration was enhanced. The hydration products aggregated in the pore structures, and the pores were blocked gradually which ended the seepage of backfill. Therefore, the higher the initial temperature of backfill was, the higher the compactness of structure was and the lower the permeability was [31]. In the periods I and II of the CLS backfill cooling process, the backfill temperature was very low which restrains the hydration. The less hydration products resulted in little change of the pore structure and high permeability. While in the period III, the rising temperature of backfill enhanced the hydration, and lots of hydration heat was generated which raises the temperature further. The lots of hydration products generated and the pore structure changed a lot which resulted in high permeability. So the different seepage characteristics of the CLS functional backfill are shown in the different periods of cooling process.

3.3. Backfill Mechanics. The mechanical functions of backfill support the unsteady surrounding rock of stope and play the role of artificial pillar in exploiting the large ore body [32]. Many researches of influences of mixture ratio on tensile strength and compressive strength have been done [33, 34]. It is generally recognized that the factors of the cemented backfill strength are the cement type and addition material, filling concentration, tailings chemical composition and gradation, mixing time, curing age, and condition [35]. In the case of CLS filling slurry, both the solid properties of ice and the adhesive of ice surface enhance the strength. The researches of concrete proved this. Suzuki et al. [36] developed the method of using crushed ice instead of water for mixing concrete which having the two times of strength than conventional concrete. Furthermore, by considering the negative effect of hydration heat, the casting temperature of concrete was limited by standards of construction. For example, the upper limit of casting temperature is $18^{\circ} \mathrm{C}$ [37]. This limit was set to decrease the thermal stress and the concrete cracks resulted from nonuniform temperature 


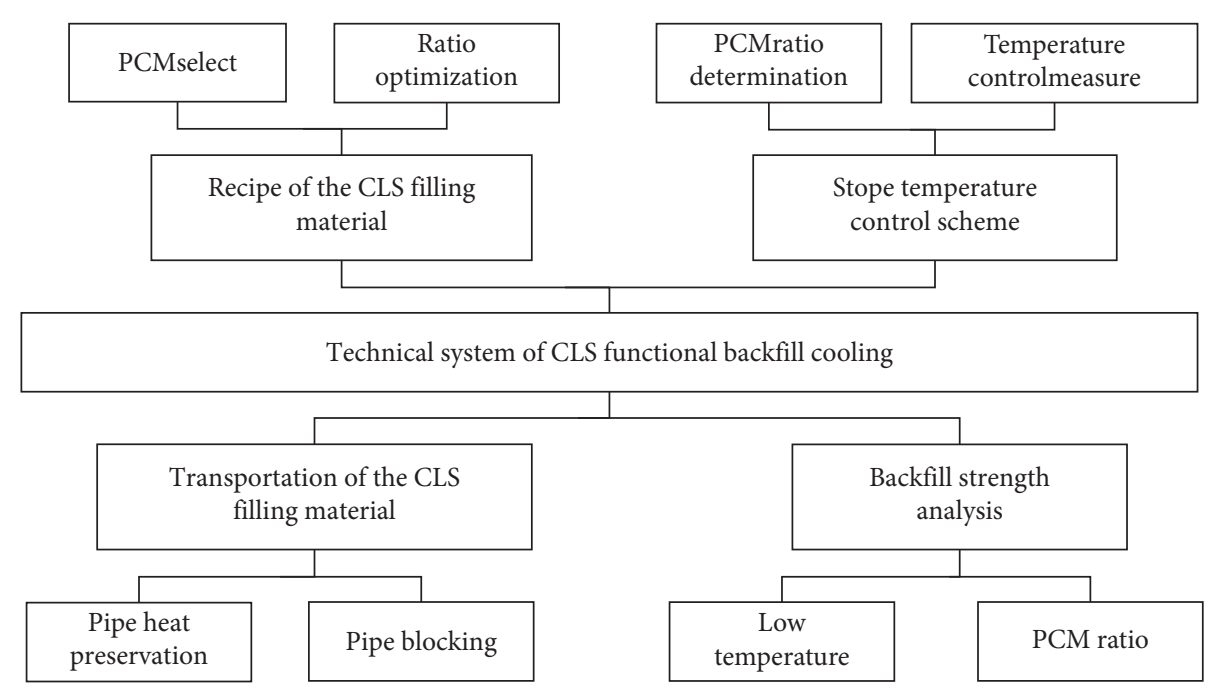

FIgUre 4: The technical system of CLS functional backfill cooling.

distribution of material. However, the low initial temperature could decrease the strength by retaining hydration. Liu et al. [38] studied the cementing waste rock strength of a gold mine in cold region through experiment. It was shown that the curing temperature had obvious impact on strength. The strength increased by rising curing temperature especially in early age than in later period.

According to the analysis above, in the early age, there are two different effects of low temperature on CLS backfill strength, while in the later period, the temperature rises and its impact fades away.

\section{Technical System of CLS Functional Backfill Cooling}

The implement of CLS functional backfill involves the recipe of the filling material, transport scheme of filling material, stope temperature controlling scheme, and strength analysis of backfill. According to the aspects above, the technology system of CLS functional backfill was built (Figure 4).

4.1. The Recipe of the CLS Filling Material. CLS filling material is a mixture of waste rock and tailings, PCM with cold load and storage function, and cemented material. The selection of CLS PCM and the optimization of the ratio of components are the two key issues.

The selection of CLS PCM is based on the principles of high cold load density, nonhazardous, stable in chemical composition, accessible, and low material cost and cold storage cost. The theory analysis of selecting ice as CLS PCM was discussed above. Whether the other PCM material could be applied should be analyzed and identified ulteriorly.

There are three ways to optimize the ratio of components. First, orthogonal experimental design which used partial experiments instead of overall experiments is the most common method. It is the main method of factorial design. Wu et al. [39] applied this method on design of cemented filling material and obtained the optimized ratio of components by range analysis. Second, uniform experiment design: this method considers how to distribute the design points evenly within the scope of the experiment in order to ensure the experiment points have the statistical properties of uniform distribution. Hu et al. [40] used this method and got the optimized ratio of filling slurry components. Third, formulation experiment design: this method is a special mixture experiment design. Yang et al. [41] applied this method and built a fitting model of the strength with the ratio of every component for cement-steel slag-mineral slag ternary material. The research of the optimized ratio of CLS functional backfill material should consider the flow characteristics and mechanics and potential of heat absorption.

4.2. The Transportation of the CLS Filling Material. Besides the conventional transportation problems, the pipe heat preservation and pipe blocking problem in CLS filling material transportation system should be paid attention to.

In order to decrease the cold loss in the CLS filling material transportation process, the PCM with suitable phase change temperature could be selected which changed the phase only under the temperature condition of goaf. However, the goaf temperature changes variably with mining depth, and it is close to transportation temperature. So this method is hard to realize. The feasible method is retrofitting the heat preservation layer to the filling slurry pipe. Considering the requirement of fire proofing of mine, glass wool or rock wool could be used as heat preservation layer material.

The pipe blocking problem of CLS filling material is more serious than conventional filling material because of the PCM. Taking water, for example, some water in the slurry may freeze under undercooling condition. The waste rock and tailings in slurry contribute to the phase change owing to them providing a lot of surface for crystal nucleation. Furthermore, the ice surface melts slightly. When it contacts with other surface, cementation occurs under undercooling condition and large size ice particles are 
created. To avoid the ice block problem, three intervention measures should be taken: (1) Optimal design of the slurry transportation pipe; (2) Initiative melting based on monitoring ice block location; and (3) Interior wall of pipe coating for decreasing interfacial energy and improving flow capacity of the pipe [42].

4.3. Stope Temperature Control Scheme. By insuring the stope temperature meets the requirement in a whole period of stope exploiting, there are two aspects should be paid attention to. First, the total cooling load should be calculated according to the cold load condition of stope and total exploiting time of a layer so that the PCM ratio could be determined. Second, the influence factors of stope temperature should be analyzed comprehensively to obtain the temperature field change trend. And the temperature control scheme was established on the trend.

The CLS functional backfill cooling involves the backfill region, ore body region, and stope region. Heat transfers between three regions. The cooling capacity of CLS backfill decayed which results in cold discharge rate changes in a cooling period. So, it is need to weaken heat transfer in the initial stage of a cooling period and enhance heat transfer in the later stage. For weakening heat transfer, thermal conductivity resistance could be increased through laying temporary thermal insulation layer on the ground of the stope at the preliminary period of cooling. For enhancing heat transfer, the convective heat transfer coefficient between air and ground of the stope could be increased through adding local forced heat convection by fans. By taking this scheme, the stope temperature could be kept nearly constant.

4.4. Backfill Strength Analysis. The backfill strength is related to density, porosity, cementitious material ratio, and so on. On account of the PCM, the composition proportions of CLS backfill differs from conventional backfill a lot. And it would influence the backfill strength. There are two aspects for analysis of CLS backfill strength: low-temperature influence and composition proportion of PCM influence. Analogy, experience formulas, physical simulation, numerical analysis, and probabilistic method could be used on this research.

\section{Conclusions}

According to this study, four conclusions are underlined as follows:

(i) The concept of the CLS functional backfill cooling method was proposed. The cooling process and principle was introduced by taking the upper ward filling method as an example.

(ii) The Conventional mine cooling method was summarized, and the advantages of the CLS backfill cooling method on investment, running cost, and safety were listed. A conclusion that this method is very suitable for high temperature deep mine cooling was achieved by comparison.

(iii) Theories of CLS functional backfill cooling were discussed. Heat transfer, flow mechanics, and backfill mechanics which involved in this method were analyzed through the literature research.

(iv) The technical system of CLS functional backfill cooling was built which consists of the recipe and transportation of the CLS filling material, stope temperature control scheme, and backfill strength analysis.

This research indicated the direction of research and development of CLS functional backfill cooling.

\section{Conflicts of Interest}

The authors declare that there are no conflicts of interest regarding the publication of this paper.

\section{Authors' Contributions}

Mei Wang designed the research framework and wrote the manuscript. Lang Liu conceived the new method. Xiaoyan Zhang collected the data of literatures. Liu Chen, Bo Zhang, and Changfa Ji analyzed the feasibility of the new method.

\section{Acknowledgments}

The authors are grateful for the support provided by the Natural Science Basic Research Plan in Shaanxi Province (no. 2018JQ5183) through the Program "The deep mine cooling mechanism and the temperature controlling effect and efficiency of cold storage PCM backfill," the Foundation of Shaanxi Provincial Department of Education (no. 18JK528), and the National Natural Science Foundation of China (nos. 51674188, 51504182, 51404191, and 51504188).

\section{References}

[1] H. P. Xie, "Research framework and anticipated results of deep rock mechanics and mining theory," Advanced Engineering Sciences, vol. 49, pp. 1-16, 2017.

[2] P. Y. Guo, Characteristics of Geothermal Field of Deep Mine and Its Heat Damage Control in China, China University of Mining and Technology, Beijing, China, 2010.

[3] X. B. Li, J. Zhou, S. F. Wang, and B. Liu, "Review of exploitation methods for deep solid resources," Chinese Journal of Nonferrous Metals, vol. 27, pp. 1236-1262, 2017.

[4] B. You, C. Wu, J. Li, and H. M. Liao, "Physiological responses of people in working faces of deep underground mines," International Journal of Mining Science and Technology, vol. 24, no. 5, pp. 683-688, 2014.

[5] M. C. He and P. Y. Guo, "Deep rock mass thermodynamic effect and temperature control measures," Chinese Journal of Rock Mechanics and Engineering, vol. 32, no. 12, pp. 23772393, 2013.

[6] M. C. He and M. Xu, "Research and development of hems cooling system and heat-harm control in deep mine," Chinese Journal of Rock Mechanics and Engineering, vol. 27, no. 7, pp. 1353-1361, 2008. 
[7] F. X. Bu, M. G. Zhang, X. X. Meng, and Q. R. Liu, “Application of natural ice cold storage in mine cooling," Coal Technology, vol. 34, pp. 158-160, 2015.

[8] J. H. Shi, S. Xin, and S. J. Yu, "The feasibility analysis of natural ice-making cooling technology in mine cooling," Industrial Safety and Environmental Protection, vol. 37, pp. 22-26, 2011.

[9] G. E. du Plessis, L. Liebenberg, and E. H. Mathews, "Case study: the effects of a variable flow energy saving strategy on a deep-mine cooling system," Applied Energy, vol. 102, pp. 700-709, 2013.

[10] S. A. Ghoreishi-Madiseh, F. Hassani, and F. Abbasy, "Numerical and experimental study of geothermal heat extraction from backfilled mine stopes," Applied Thermal Engineering, vol. 90, pp. 1119-1130, 2014.

[11] A. Sharma, V. V. Tyagi, C. R. Chen, and D. Buddhi, "Review on thermal energy storage with phase change materials and applications," Renewable and Sustainable Energy Reviews, vol. 13, no. 2, pp. 318-345, 2009.

[12] S. M. Yang, Heat Transfer, Higher Education Press, Beijing, China, 4th edition, 2011.

[13] G. Y. Fang, New Practical Technologies of Cold Storage in Air Conditioning Engineering, Post and Telecom Press, Beijing, China, 2000.

[14] J. H. Liu, R. H. Liu, C. H. Wang, and Y. N. Liang, "Thermodynamic test of $\mathrm{Na}_{2} \mathrm{SO}_{4} \cdot 10 \mathrm{H}_{2} \mathrm{O}$ phase change energy storage system," Energy Conservation, vol. 9, pp. 13-17, 2007.

[15] W. B. Tao and R. H. Xie, "Research and development of organic phase change materials for cool thermal energy storage," Journal of Refrigeration, vol. 37, pp. 52-59, 2016.

[16] D. Y. Jia, Y. X. Du, Y. P. Yuan, and B. Y. Cheng, "An organic phase change material applied in cold storage of air conditioner," China Patent, CN101220259, 2008.

[17] L. Royon and G. Guiffant, "Heat transfer in paraffin oil/water emulsion involving supercooling phenomenon," Energy Conversion and Management, vol. 42, no. 18, pp. 2155-2161, 2001.

[18] P. Doron and D. Barnea, "A three-layer model for solid- liquid flow in horizontal pipes," International Journal of Multiphase Flow, vol. 19, no. 6, pp. 1029-1043, 1993.

[19] X. D. Liu and X. Y. Chen, "Fluid mechanics analysis and velocity calculation of filling slurry pipeline transportation," Modern Mining, vol. 499, pp. 28-31, 2010.

[20] Q. Wang, X. Zhang, and E. Z. Zhang, "The flow characteristics of ice slurry and its application research," in Proceedings of the 2011 Annual Conference of Shanghai Institute of Refrigeration, Shanghai, China, May 2011.

[21] X. Y. Zhang, Research on Key Technologies of Cooling Source of Mine Cooling System for Ultra-Deep Mines, Wuhan University of Technology, Wuhan, China, 2009.

[22] J. Guilpart and L. Fournasion, "Experimental study and calculation method of transport characteristics of ice slurries," in Proceedings of the First Workshop on Ice Slurries, Paris, France, 1999.

[23] D. Wang, Z. L. Xu, and Q. H. Meng, "Effect factors and calculating analysis of critical flow velocity in slurry pipeline transportation," Pipeline Technique and Equipment, vol. 6, pp. 1-2, 2004.

[24] X. J. Fei, Hydraulics of Slurry with Granular Material Transportation, Tsinghua University Press, Beijing, China, 1994.

[25] T. Hansen, M. Radosevic, and M. Kauffeld, Behavior of Ice Slurry in Thermal Storage Systems, ASHRAE, Atlanta, GA, USA, 2002.
[26] P. Pronk, T. M. Hansen, C. A. Infante Ferreira, and G. J. Witkamp, "Time-dependent behavior of different ice slurries during storage," International Journal of Refrigeration, vol. 28, no. 1, pp. 27-36, 2005.

[27] P. Pronk, C. A. Infante Ferreira, and G. J. Witkamp, "Effects of long-term ice slurry storage on crystal size distribution," in Proceedings of the Fifth Workshop on Ice Slurries of the International Institute of Refrigeration, Stockholm, Sweden, May 2002.

[28] H. Kanetoshi and K. Ken, "A method for measuring ice slurry particle agglomeration in storage tanks," in Proceedings of the ASHRAE Transactions 106 CPA, pp. 117-123, Honolulu, HI, USA, 2002.

[29] A. X. Xu, Z. Q. Liu, T. L. Zhao, and X. X. Wang, "Factors influencing dynamics evolution of ice crystals during ice slurry storage," Journal of Central South University (Science and Technology), vol. 46, pp. 3138-3144, 2015.

[30] Y. X. Mao, L. X. Ding, and C. C. Feng, "Study on permeability problems of filling in the sandfill mining stope," Metal Mine, vol. 446, pp. 27-30, 2013.

[31] D Wu, G. H. Sun, and G. Huang, "Experimental and simulation study on seepage characteristics of cemented tailings backfill," Journal of Central South University (Science and Technology), vol. 46, pp. 1050-1057, 2015.

[32] L. J. Guo and X. C. Yang, "Study on mechanics stability of cemented backfill for mine deep stope," Mining and Metallurgy, vol. 17, pp. 10-13, 2008.

[33] R. H. Cao and H. Lin, "Experimental and numerical study of failure behavior and energy mechanics of rock-like materials containing multiple joints," Advances in Materials Science and Engineering, vol. 2017, Article ID 6460150, 17 pages, 2017.

[34] H. Lin, W. Xiong, and Q. X. Yan, "Modified formula for the tensile strength as obtained by the flattened Brazilian disc test," Rock Mechanics and Rock Engineering, vol. 49, no. 4, pp. 1579-1586, 2016.

[35] Z. X. Liu, Mechanics of High Tailings Backfill and Nonlinear Optimal Design in Deep Mining, Central South University, Changsha, China, 2005.

[36] T. Suzuki, K. Takiguchi, and H. Hotta, "Characteristics of concrete produced by new method using small ice flakes," in Proceedings of the JCI Conference, Tokyo, Japan, June 1986.

[37] G. C. Wei, "Cement temperature control of three gorges hydro project," Northwest Hydroelectric, vol. 3, pp. 38-40, 2001.

[38] C. Liu, B. Han, W. Sun, J. X. Wu, S. Yao, and H. Y. Hu, "Experimental study of strength of backfilling of cemented rock debris and its application under low temperature condition," Chinese Journal of Rock Mechanics and Engineering, vol. 34, pp. 139-147, 2015.

[39] G. Wu, Y. P. Zhang, and Z. K. Zeng, "The design of optimized strength parameters of cementing filling uses orthogonal test method," Mining Engineering, vol. 6, pp. 24-26, 2010.

[40] X. Y. Hu, L. Liu, G. H. Li, and L. J. Guo, "Ratio optimization on filling slurry of mine based on uniform experiments," Mining Research and Development, vol. 3, pp. 7-12, 2015.

[41] C. Yang, D. Y. Lu, Z. Z. Xu, and Y. G. Wan, "Optimization of the mix proportion in the ternary cementitious system of cement-steel slag-gobs based on mixture design," Bulletin of the Chinese Ceramic Society, vol. 34, pp. 2107-2112, 2015.

[42] R. Wu, The Analysis and Experiment on Dynamic Ice Slurry Generation Based on Supercooled Method, Huazhong University of Science and Technology, Wuhan, China, 2006. 


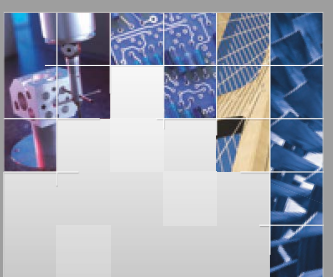

\section{Enfincering}
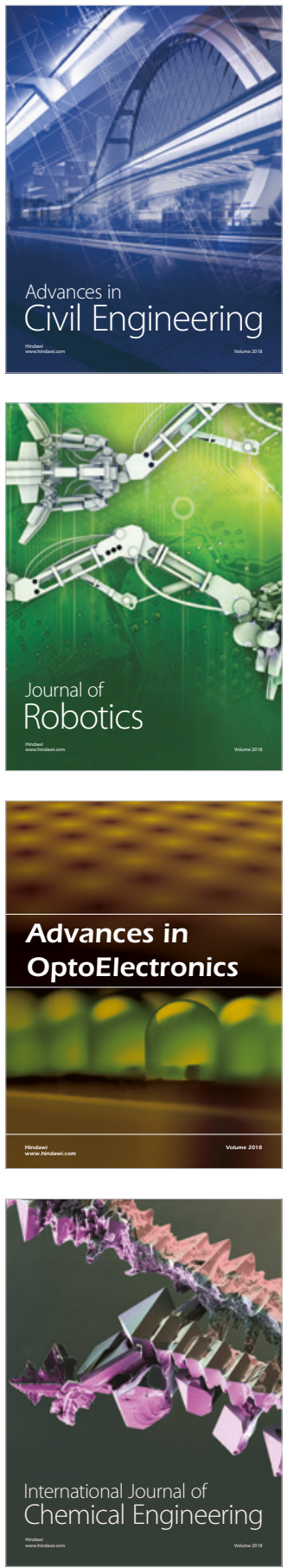

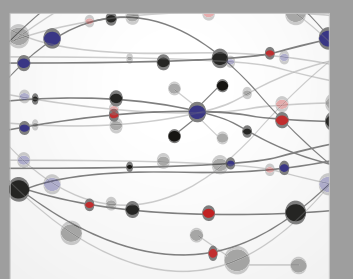

\section{Rotating \\ Machinery}

The Scientific World Journal

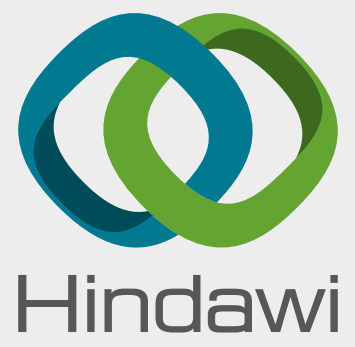

Submit your manuscripts at

www.hindawi.com
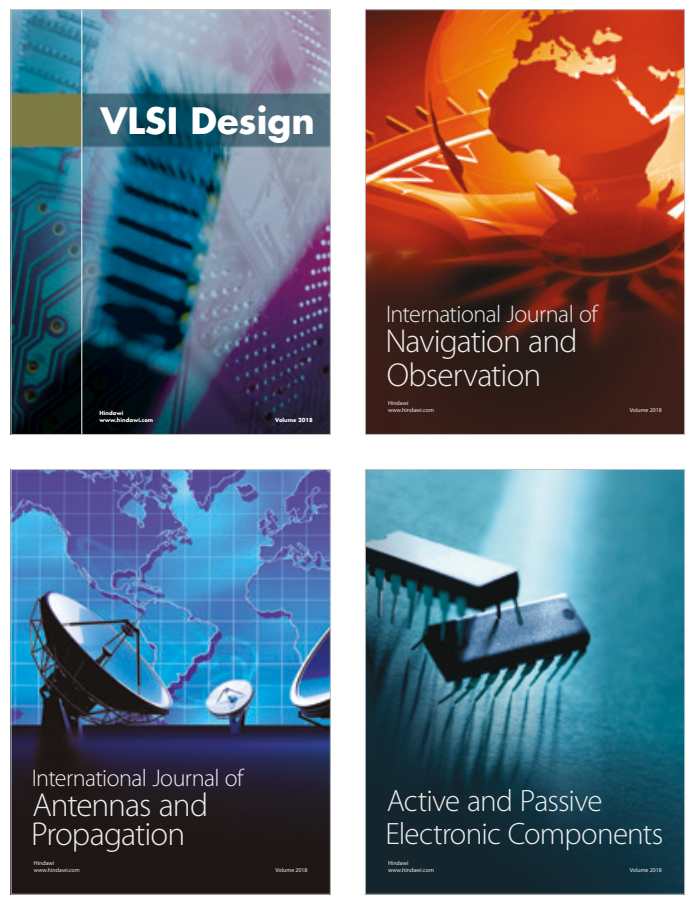
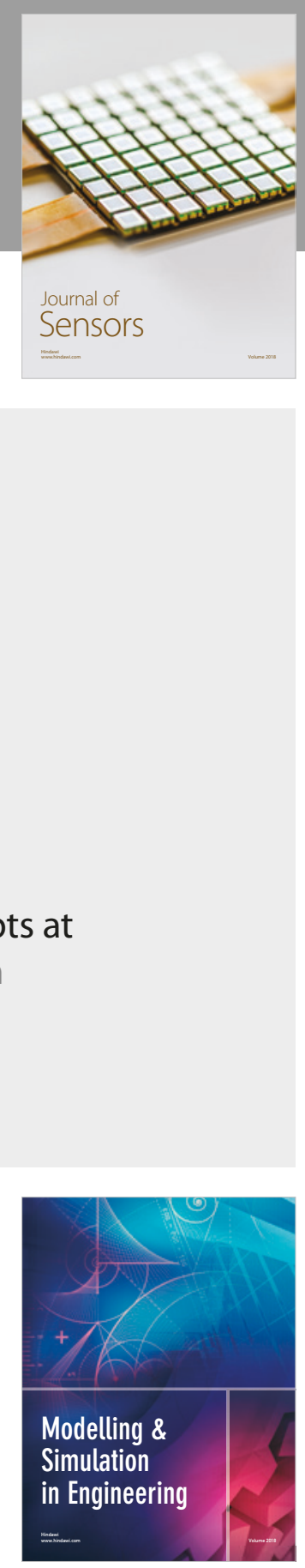

\section{Advances \\ Multimedia}
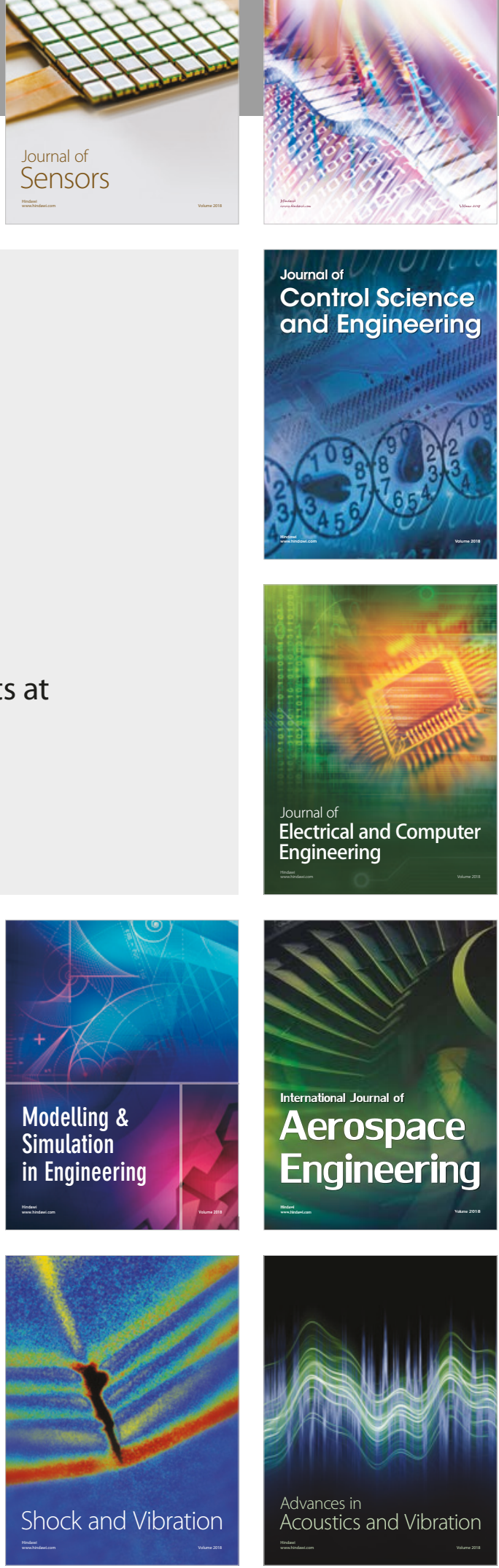